\title{
BEST-FIT PARAMETERS OF MIXED DARK MATTER MODEL FROM ABELL-ACO POWER SPECTRA AND MASS FUNCTION
}

\author{
B. Novosyadlyj \\ Astronomical Observatory of the Ivan Franko State University of Lviv, \\ 8 Kyrylo i Mefodii Str., Lviv, UA-290005, Ukraine
}

(Received December 25, 1998)

\begin{abstract}
The possibility of determining MDM model parameters on the basis of observable data on the Abell-ACO power spectrum and mass function is analysed. It is shown that spectrum area corresponding to these data is sensitive enough to such MDM model parameters as neutrino mass $m_{\nu}$, number species of massive neutrino $N_{\nu}$, baryon content $\Omega_{b}$ and Hubble constant $h \equiv H_{0} / 100 \mathrm{~km} / \mathrm{s} / \mathrm{Mpc}$. The $\chi^{2}$ minimization method was used for their determination. If all these parameters are under searching then observable data on the Abell-ACO power spectrum and mass function prefer models which have parameters in the range $\Omega_{\nu}(\sim 0.4-0.5)$, low $\Omega_{b}(\leq 0.01)$ and $h$ $(\sim 0.4-0.6)$. The best-fit parameters are as follows: $N_{\nu}=3, m_{\nu}=4.4 \mathrm{eV}, h=0.56, \Omega_{b} \leq 0.01$. The high- $\Omega_{b} \sim 0.4-0.5$ solutions are obtained when mass of neutrino is fixed and $\leq 3 \mathrm{eV}$.

To explain the observable excessive power at $k \approx 0.05 h / M p c$ the peak of Gaussian form was introduced in primordial power spectrum. Its parameters (amplitude, position and width) were determined along with the MDM model parameters. It decreases $\chi^{2}$, increases the bulk motions, but does not change essentially the best-fit MDM parameters.

It is shown also that models with the median $\Omega_{\nu} \sim 0.2-0.3\left(m_{\nu} \sim 2.5, N_{\nu} \sim 2-3\right)$ and $\Omega_{b}=0.024 / h^{2}$, which match constraints arising from cosmological nucleosynthesis and high redshift objects, are not ruled out by these data $\left(\Delta \chi^{2}<1\right)$.
\end{abstract}

Key words: Large Scale Structure, Abell-ACO power spectrum, mass function, Mixed Dark Matter models, initial power spectra, best-fit cosmological parameters.

PACS numbers: 98.80.Ft, 98.80.Es, 98.65.Dx

\section{INTRODUCTION}

The observable data on large scale structure of the Universe obtained during last years and coming from current experiments and observational programs give a possibility to determine more exactly the parameters of cosmological models and the nature of the dark matter. Up till now the most certain data are about the largest scale inhomogeneities of the current particle horizon of the order of $\sim 7000 h^{-1} \mathrm{Mpc}\left(h \equiv H_{0} / 100 \mathrm{~km} / \mathrm{s} / \mathrm{Mpc}\right.$, $H_{0}$ is today Hubble constant) which are obtained from the study of all-sky temperature fluctuations of cosmic microwave background $(\mathrm{CMB})$ with $\sim 10^{\circ}$ angular resolution by the space experiment COBE $[44,2,3]$. According to them the primordial power spectrum of density fluctuations is approximately scale invariant $P_{p i}(k)=A k^{n}$ with $n=1.1 \pm 0.2$ that well agrees with the predictions of standard inflation model of the Early Universe ( $n=1, \Omega_{0}=1$ ). Besides, they most certainly determine the amplitude of a linear power spectrum (or normalization constant $A$ ) which does not depend on any transition processes, nonlinearity effects and other phenomena connected with the last stages of large scale structure formation. On the contrary, the CMB temperature fluctuations at degree and sub-degree scales as well as the space distributions of the cluster of galaxies, galaxies, quasars, Lyman- $\alpha$ clouds, etc. are defined by those processes and also depend essentially on the nature of the dark matter. Theoretically it is taken into account by introducing the transfer function $T(k)$ which transforms the primordial (post-inflation spectrum) into the postrecombination (initial) one $-P(k)=P_{p i}(k) T^{2}(k)$, which defines all characteristics of the large scale structure of the Universe. The transfer function depends also on the curvature of the Universe or the present energy density in units of critical density, $\Omega_{0}$, vacuum energy density or cosmological constant $\Omega_{\Lambda}$, content of baryons $\Omega_{b}$, and values of the Hubble constant. The theory of a large scale structure formation is so far advanced today that all these dependencies can be accurately calculated for the fixed model by public available codes (e.g. CMBfast one by [43]). The actual problem now is the determination of the nature of the dark matter and the rest of the above mentioned parameters by means of comparison of theoretically predicted and observable characteristics of the large scale structure of the Universe.

As most advanced candidates for the dark matter are cold dark matter (CDM), particles like axions, hot dark matter (HDM), particles like massive neutrinos with $m_{\nu} \sim 1-20 \mathrm{eV}$ and baryon low luminosity compact objects. The last ones can not dominate as it results from the cosmological nucleosynthesis constraints $\left(\Omega_{b} h^{2} \leq\right.$ $0.024,[46,45,42])$ and observation of microlensing events in the experiments like MACHO, DUO, etc. The pure HDM model conflicts with the existence of high redshift objects, the pure CDM one, on the contrary, overpredicts them. Therefore mixed dark matter (MDM) model (CDM+HDM+baryons) with $\Omega_{H D M} \equiv \Omega_{\nu} \leq 0.3$ looks more viable. The advantage of these models is a small 
number of free parameters. But today it is understood already that models with the minimal number of free parameters, such as a standard cold dark matter (sCDM, one parameter) or a standard cold plus hot mixed dark matter (sMDM, two parameters) only marginally match the observable data. A better agreement between theoretical predictions and observable data is achieved in the models with a larger number of free parameters (tilted CDM, open CDM, CDM or MDM with the cosmological term, see review in [48] and references therein).

The oscillations of solar and atmospheric neutrinos registered by SuperKamiokande experiment show that the difference of rest masses between $\tau$ - and $\mu$ neutrinos is $0.02<\Delta m_{\tau \mu}<0.08 \mathrm{eV}[14,38]$. It also gives a lower limit for the mass of neutrino $m_{\nu} \geq|\Delta m|$ and does not exclude models with cosmologically significant values $\sim 1-20 \mathrm{eV}$. Therefore, at least two species of neutrinos can have approximately equal masses in this range. Some versions of elementary particle theories predict $m_{\nu_{e}} \approx m_{\nu_{\tau}} \approx 2.5 \mathrm{eV}$ and $m_{\nu_{\mu}} \approx m_{\nu_{s}} \sim 10^{-5} \mathrm{eV}$, where $\nu_{e}, \nu_{\tau}, \nu_{\mu}$ and $\nu_{s}$ denote the electron, $\tau-, \mu-$ and sterile neutrinos accordingly (e.g. [4]). The strongest upper limit for the neutrino mass comes from the data on a large scale structure of our Universe: $\sum_{i} m_{\nu_{i}} / 93 h^{2} \leq 0.3$ $[20,7,41,49,31,35,29,48]$, that for $h=0.8$ (the upper observable limit for $h$ ) gives $\sum_{i} m_{\nu_{i}} \leq 18 \mathrm{eV}$. It is interesting that the upper limit for the mass of electron neutrino obtained from supernova star burst SN1987A neutrino signal is approximately the same $m_{\nu_{e}} \leq 20 \mathrm{eV}$.

Is it possible to find the best fit neutrino mass from experimental data on a large scale structure of the Universe? The problem is that it must be determined together with other large number uncertain parameters such as $h, \Omega_{0}, \Omega_{b}$, etc. Here we study the possibility of finding them by $\chi^{2}$ minimization method. Realization of such a task became possible in principle after the appearance in literature of accurate analytical approximations of transfer function for mixed dark matter model in at least 4-dimension space of the above mentioned cosmological parameters $T\left(k ; \Omega_{b}, m_{\nu}, N_{\nu}, h\right)[12,33]$. That is why that even CMBfast codes are too bulky and slow yet for searching the cosmological parameters by the methods of minimization of $\chi^{2}$, like Levenberg-Marquardt one (see [37]).

The next problem is a choice of the observable data suitable for the solution of this task. They must be enough accurate, sensitive to those parameters and not too dependent on the model assumptions about the formation and nature of objects. The most sensitive to the presence of neutrino component are scales of order and smaller of its free-streaming (or Jeans) scale $k \geq k_{J}(z)=8\left(\frac{m_{\nu}}{10 e V}\right) / \sqrt{1+z} h^{-1} \mathrm{Mpc}$ because perturbations at these scales are suppressed and it is imprinted in the transfer function of the HDM component. At $z \sim 0$ for cosmologically significant neutrino masses it is approximately galaxy clusters scale. The power spectrum reconstructed from space distributions of galaxies is distorted significantly by nonlinearity effects the accounting of which is model dependent [34]. The models of the formation of smaller scale structures or high red- shift objects (e.g. Lyman- $\alpha$ damped systems, Lyman $-\alpha$ clouds, quasars etc.) contain the additional assumptions and parameters which makes their using rather problematic in such an approach. The CMB temperature anisotropy at subdegree angular scales (first and second acoustic peaks) has minimal additional assumptions (e.g. secondary ionization) but its sensibility to the presence of neutrino component is low $(\leq 10 \%,[8])$. These data are sensitive and suitable for determination by $\chi^{2}$ minimization methods other set of parameters such as tilt of primordial spectrum $n, \Omega_{0}, h, \Omega_{b}, \Omega_{\Lambda}$ or/and parameters of scaling seed models of structure formation (see [28,9]).

The data on Abell-ACO power spectrum and function mass of rich clusters seem to be suitable for determining the best fit values of $m_{\nu}$ and $N_{\nu}$ because they do not depend on the above mentioned additional assumptions.

The data on rich clusters power spectrum [10] were used in $[1,13]$ for analyzing $\sim 100 h^{-1} \mathrm{Mpc}$ clustering. The first collaboration group tried to explain the narrow peak in the power spectrum at $\sim 100 h^{-1} \mathrm{Mpc}$ scale by baryonic acoustic oscillations in low- and high $-\Omega_{0}$ models $\left(\Omega_{0}=\Omega_{C D M}+\Omega_{b}\right)$. In both cases such an approach needs very high content of baryons $\Omega_{b}(>0.3)$, that is essentially out of the cosmological nucleosynthesis constraints. The second one has shown that this feature is in agreement with Saskatoon data [30] on $\Delta T / T$ power spectrum at subdegree angular scales. They have concluded that these data prefer models with built-in scale in the primordial power spectrum which can be generated in the more complicated inflation scenario (e.g. double one).

For reducing the number of free parameters we restrict ourselves to analysis within the framework of the matter dominated Universe and standard inflation scenario: $\Omega_{0} \equiv \Omega_{C D M}+\Omega_{\nu}+\Omega_{b}=1, n=1$ without the tensor mode of cosmological perturbations. The free parameters in our task will be baryon content $\Omega_{b}$, dimensionless Hubble constant $h$, neutrino mass $m_{\nu}$, and number species of neutrinos with equal masses $N_{\nu}$.

The outline of this paper is as follows: the observable data which will be used here are described in Section II. The method of determination of parameters and its testing are described in Sect. III. Results of best fit finding of parameters under different combination of free and fixed ones are presented in Sect. IV. Discussion of results and conclusions are given in Sect. V and VI accordingly.

\section{EXPERIMENTAL DATA SET}

The most favorable data for the search of best fit cosmological parameters are real power spectrum reconstructed from redshift-space distribution of Abell- ACO clusters of galaxies $[10,40]$. It is biased linear spectrum reliably estimated for $0.03 \leq k \leq 0.2 h / M p c$ whose position of maximum $\left(k_{\max } \approx \overline{0} .05 \bar{h} / M p c\right)$, inclination before and after it are sensitive to baryon content $\Omega_{b}$. Hubble constant $h$, neutrino mass $m_{\nu}$ and number species of massive neutrinos $N_{\nu}$ (see Fig. 1-4). Here in numerical calculations the data of last estimation of power spec- 


\section{B. NOVOSYADLYJ}

trum by [40] will be used. All the sources of systematic and statistical uncertainties as well as window function and differences between Abell and ACO parts of sample have been accurately taken into account there. The values of the Abell-ACO power spectrum for 13 values of $k$ $\tilde{P}_{A+A C O}\left(k_{j}\right)(j=1,13)$ and their $1 \sigma$ errors are presented in table 1 and are shown in figures $5-7$.

Other observable data which will be used here are constraints of amplitude of the fluctuation power spectrum at cluster scale derived from cluster mass and $X-$ ray temperature functions. It is usually formulated as a constraint for density fluctuations in top-hat sphere of $8 h^{-1} \mathrm{Mpc}$ radius, $\sigma_{8}$, which can be easy calculated for the given initial power spectrum $P(k)$ :

$$
\sigma_{8}^{2}=\frac{1}{2 \pi^{2}} \int_{0}^{\infty} k^{2} P\left(k ; \Omega_{b}, h, m_{\nu}, N_{\nu}\right) W^{2}(8 k / h) d k
$$

where $W(x)=3(\sin x-x \cos x) / x^{3}$ is Fourier transformation of top-hat window function. The different collaboration groups gave similar results which are in the range of $\tilde{\sigma}_{8} \sim 0.5-0.7$. The new optical determination of the mass function of nearby galaxy clusters [16] gives median values: $\tilde{\sigma}_{8}=0.60 \pm 0.04[17]$. It matches very well the cluster $X$-ray temperature function [50]. For taking into account the data of other authors I shall be more conservative and will use it with $3 \sigma$ error bars instead of $1 \sigma$ one. But, as we will see, it does not rule out predicted $\sigma_{8}$ value from the $1 \sigma$ limit of the observable one by [16] for best fit parameters determined here.

\begin{tabular}{|cccc|}
\hline No & $k_{j}$ & $\tilde{y}_{j}$ & $\Delta \tilde{y}_{j}$ \\
\hline 1 & 0.030 & $9.312 \cdot 10^{4}$ & \pm 59723.65 \\
2 & 0.035 & $1.037 \cdot 10^{5}$ & \pm 65488.2 \\
3 & 0.040 & $1.039 \cdot 10^{5}$ & \pm 58014.15 \\
4 & 0.047 & $1.258 \cdot 10^{5}$ & \pm 51005.75 \\
5 & 0.054 & $1.448 \cdot 10^{5}$ & \pm 68638.6 \\
6 & 0.062 & $1.016 \cdot 10^{5}$ & \pm 39184.6 \\
7 & 0.072 & $8.098 \cdot 10^{4}$ & \pm 25179.7 \\
8 & 0.083 & $5.444 \cdot 10^{4}$ & \pm 21925.45 \\
0 & 0.096 & $5.303 \cdot 10^{4}$ & \pm 24914.75 \\
10 & 0.11 & $3.853 \cdot 10^{4}$ & \pm 13344.5 \\
11 & 0.13 & $2.031 \cdot 10^{4}$ & \pm 8546.35 \\
12 & 0.15 & $2.039 \cdot 10^{4}$ & \pm 9804.3 \\
13 & 0.17 & $1.691 \cdot 10^{4}$ & \pm 9383.21 \\
14 & $\sigma_{8}$ & 0.60 & \pm 0.12 \\
\hline
\end{tabular}

Table 1. Experimental data set.

The COBE 4-year data will be used here for normalization of power spectra. A useful fit for them is the amplitude of density perturbation of the horizon crossing scale $\delta_{h}$, which for a flat model with the $n=1$ equals $\delta_{h}=1.94 \cdot 10^{-5}[27,6]$. Taking into account the definition of $\delta_{h}[27]$ and the power spectrum, the normalization constant $A$ is calculated as

$$
A=2 \pi^{2} \delta_{h}^{2}(3000 / h)^{4} M p c^{4} .
$$

\section{METHOD AND ITS TESTING}

The Abell-ACO power spectrum is connected with matter one by means of the cluster biasing parameter $b_{c l}$ :

$$
P_{A+A C O}(k)=b_{c l}^{2} P\left(k ; \Omega_{b}, h, m_{\nu}, N_{\nu}\right)
$$

For fixed parameters $\Omega_{b}, h, m_{\nu}, N_{\nu}$ and $b_{c l}$ the values of $P_{A+A C O}\left(k_{j}\right)$ are calculated for the same $k_{j}$ as in table 1 and $\sigma_{8}$ according to (1). Let's denote them by $y_{j}(j=1, \ldots, 14)$, where $y_{1}, \ldots, y_{13}$ correspond $P_{A+A C O}\left(k_{1}\right), \ldots, P_{A+A C O}\left(k_{13}\right)$, and $y_{14}$ is $\sigma_{8}$. Their deviation from observable data set (noted by the tilde) can be described by $\chi^{2}$ :

$$
\chi^{2}=\sum_{j=1}^{14}\left(\frac{\tilde{y}_{j}-y_{j}}{\Delta \tilde{y}_{j}}\right)^{2}
$$

where $\tilde{y}_{j}$ and $\Delta \tilde{y}_{j}$ are experimental data set and their dispersion accordingly. Then parameters $\Omega_{b}, h, m_{\nu}, N_{\nu}$ and $b_{c l}$ or some part from them can be determined by minimizing $\chi^{2}$ using Levenberg-Marquard method [37]. The derivatives of predicted values on search parameters which are required by this method will be calculated numerically. The step for their calculation was experimentally assorted and is $10^{-5}$ of the values for all parameters.

The analytical approximation of MDM transfer function will be used in the form:

$$
\begin{aligned}
& T_{M D M}\left(k ; \Omega_{b}, h, m_{\nu}, N_{\nu} ; z\right) \\
& \quad=T_{C D M+b}\left(k ; \Omega_{b}, h ; z\right) D\left(k ; \Omega_{b}, h, \Omega_{\nu}, N_{\nu} ; z\right),
\end{aligned}
$$

where $T_{C D M+b}\left(k ; \Omega_{b}, h ; z\right)$ is the transfer function by [11] for CDM+baryon system ( $z$ is redshift), the correction factor for the HDM component $D(k)$ was used in the form given by [33]. It is correct in a sufficiently wide range of search parameters (for a more detailed analysis of its accuracy see in [33]). We suppose the scale invariant primordial power spectrum because the initial power spectra of MDM models now is as follows: $P_{M D M}(k)=A k T_{M D M}^{2}\left(k ; \Omega_{b}, h, m_{\nu}, N_{\nu} ; z\right)$.

The method was tested in the following way. I calculated the MDM power spectrum for the given parameters (e.g. $\Omega_{b}=0.15, \Omega_{\nu}=0.2, N_{\nu}=1, h=0.5$ ) using CMBfast code, normalized to 4-year COBE data, calculated $\tilde{\sigma}_{8}$ and interpolated $P(k)$ for the same $k_{j}(j=1, \ldots, 13)$ which are in table 1 . Then I have took cluster biasing parameter $b_{c l}=3$ and calculated model $\tilde{P}_{A+A C O}\left(k_{j}\right)$. The 'experimental' errors for them as well as for $\tilde{\sigma}_{8}$ I have suggested to be the same as relative errors from table 1. These model experimental data like the ones in table 1 were used for search of parameters $\Omega_{b}, h, \Omega_{\nu}$, and $b_{c l}$ ( $N_{\nu}$ is fixed and the same). The initial (or start) values of the parameters I have put as random deviated from the given ones. In all cases the code found all the given parameters with high accuracy. 


\section{DEPENDENCE OF DENSITY FLUCTUATIONS POWER SPECTRA AT CLUSTER SCALE ON COSMOLOGICAL PARAMETERS}

Before finding of the best-fit parameters let's look how the power spectrum of density fluctuations at cluster scale depends on search parameters. For this we leave only $b_{c l}$ as a free parameter and fix the remaining ones. In Fig. 1 such a dependence of rich cluster power spectra on $\Omega_{\nu}$ is shown for $h=0.5, \Omega_{b}=0.05$ and $N_{\nu}=1$. The r.m.s. of density fluctuations in the top-hat sphere of $8 h^{-1} \mathrm{Mpc}$ radius in models with $\Omega_{\nu}=0.1,0.2,0.3,0.4$ are $\sigma_{8}=0.93,0.81,0.75,0.71$ accordingly. The best-fit values of $b_{c l}$ are presented in the caption of Fig. 1. The deviations of the predicted rich cluster power spectra and mass function in these models from the observable ones are correspondingly $\chi^{2}=17.3,9.88,6.64,5.33$. Therefore, for the MDM model with $h=0.5, \Omega_{b}=0.05$ and $N_{\nu}=1$ Abell-ACO power spectrum and mass function prefer high $\Omega_{\nu}(\sim 0.3-0.4)$.

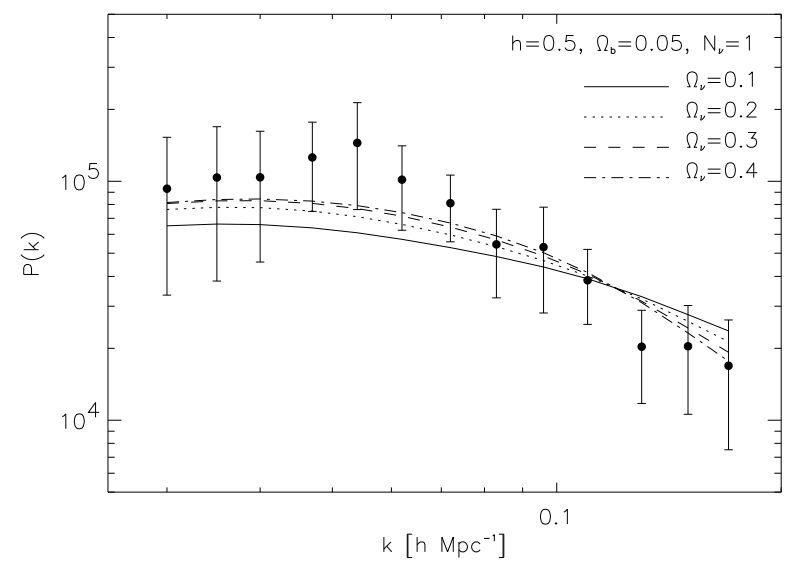

Fig. 1. The rich cluster power spectrum for MDM models with different $\Omega_{\nu}\left(N_{\nu}, \Omega_{b}\right.$ and $h$ are fixed). The filed circles are experimental Abell-ACO power spectrum by Retzlaff et al. 1997. The best-fit biasing parameters for the models with $\Omega_{\nu}=0.1,0.2,0.3,0.4$ are $b_{c l}=2.6,2.8,2.9,2.9$ accordingly.

Now we repeat the same calculations for different number species of massive neutrinos $N_{\nu}=1,2,3$ and fixed $\Omega_{\nu}=0.2$ (Fig. 2). The $\sigma_{8}$ 's for these 3 models are 0.81 , $0.73,0.68$ accordingly, the corresponding deviations of predicted rich cluster power spectra and mass functions from the observable ones respectively are $\chi^{2}=9.88,6.48$, 5.54. So, the MDM model with three species of equal mass neutrino is preferable.

In the first two cases ( $h$ fixed and equal 0.5 ) the mass of neutrino was different for differing $\Omega_{\nu}\left(N_{\nu}\right.$ fixed) and $N_{\nu}\left(\Omega_{\nu}\right.$ fixed $)$ because they are connected by relations

$$
m_{\nu}=93 \Omega_{\nu} h^{2} / N_{\nu}
$$

Let's fix the neutrino mass $\left(m_{\nu}=2.5 \mathrm{eV}\right)$, suggest that
$N_{\nu}=2$ and repeat calculations for different $h=0.5,0.6$, 0.7 . The results are shown in Fig. $3 . \sigma_{8}$ for these 3 models are following $0.71,0.98,1.24$. The $\chi^{2}$ for all points of power spectrum and $\sigma_{8}$ are $5.72,19.9$ and 42.6 accordingly. Therefore, when neutrino mass is fixed (by laboratory experiments for example) the data prefer low $h$.

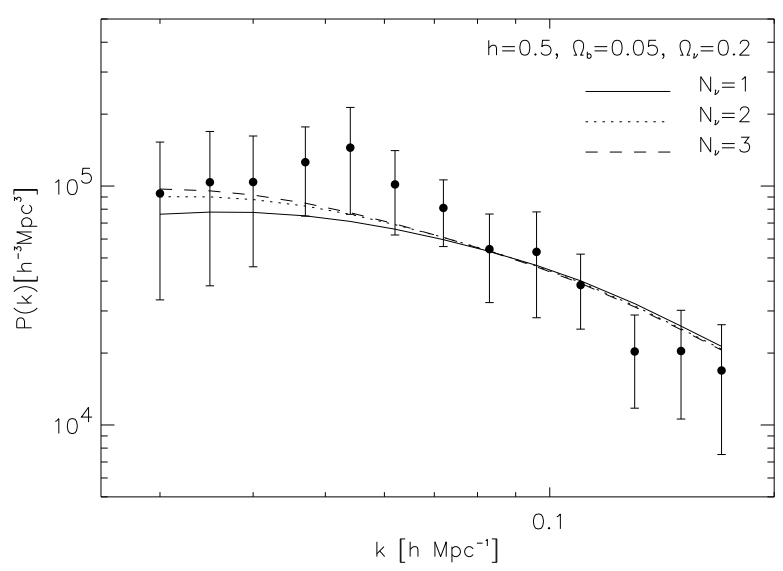

Fig. 2. The rich cluster power spectrum for MDM models with a varying number of species of massive neutrino $N_{\nu}\left(\Omega_{\nu}\right.$, $\Omega_{b}$ and $h$ are fixed). The filed circles are the same as in Fig. 1. The best-fit biasing parameters for models with $N_{\nu}=1,2,3$ are $b_{c l}=2.8,3.1,3.3$ accordingly.

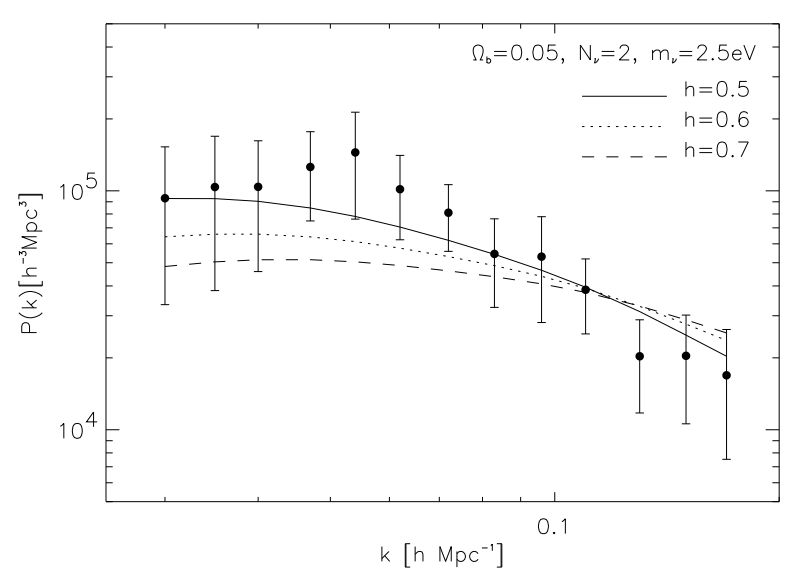

Fig. 3. The rich cluster power spectrum for MDM models with a varying $h\left(m_{\nu}, N_{\nu}\right.$ and $\Omega_{b}$ are fixed). The filed circles are the same as in Fig. 1. The best-fit biasing parameters for models with $h=0.5,0.6$ and 0.7 are $b_{c l}=3.2,2.5,2.1$ accordingly.

Similarly, one shall calculate rich cluster power spectra for different $\Omega_{b}$ when the rest of the parameters are fixed. The results for $\Omega_{b}=0.05,0.1,0.15,0.2,0.25,0.3$ are presented in Fig. 4. The corresponding $\sigma_{8}$ 's are following $0.71,0.64,0.58,0.53,0.48,0.44$, the characteristics of deviations of the predicted values from the observable ones $\chi^{2}$ for these models are 5.72, 4.28, 3.61, 3.70, 4.59, 6.39. The minimum $\chi^{2}$ is for model with $\Omega_{b}=0.15$. 


\section{B. NOVOSYADLYJ}

As we see the theoretically predicted values of the chosen data are sensitive to search parameters $m_{\nu}, N_{\nu}, \Omega_{b}$ and $h$. It is interesting now where the global minimum of $\chi^{2}$ in space of these parameters is when all or a part of them are free.

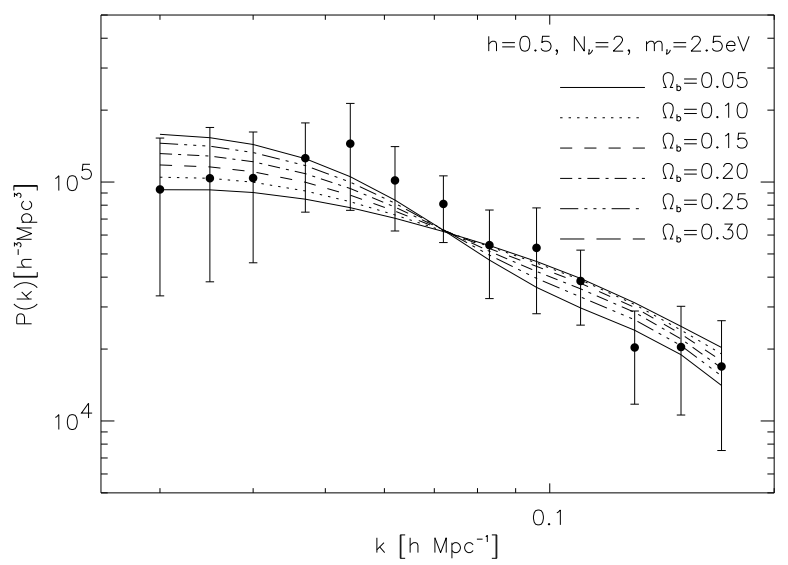

Fig. 4. The rich cluster power spectrum for MDM models with a varying $\Omega_{b}\left(m_{\nu}, N_{\nu}\right.$ and $h$ are fixed). The filed circles are the same as in Fig. 1. The best-fit biasing parameters for models with $\Omega_{b}=0.05,0.1,0.15,0.2,0.25$ and 0.3 are $b_{c l}=3.2,3.4,3.7,3.9,4.1$ and 4.3 accordingly,

\begin{tabular}{|ccccccc|}
\hline$N_{\nu}$ & $\chi^{2}$ & $\Omega_{\nu}\left(m_{\nu}\right)$ & $\Omega_{b}$ & $h$ & $b_{c l}$ & $\sigma_{8}$ \\
\hline 1 & 2.07 & $0.44(7.2)$ & 0.0006 & 0.42 & 3.49 & 0.55 \\
2 & 1.77 & $0.47(5.5)$ & 0.0014 & 0.50 & 3.37 & 0.56 \\
3 & 1.66 & $0.47(4.9)$ & 0.0021 & 0.58 & 3.29 & 0.57 \\
\hline
\end{tabular}

Table 2. Best-fit parameters of MDM models with 1, 2 and 3 sorts of massive neutrinos for Abell-ACO power spectrum by Retzlaff et al. 1997 and mass function by Girardi et al. 1998.

\section{RESULTS}

The searching of $m_{\nu}, N_{\nu}, \Omega_{b}$ and $h$ by $\chi^{2}$ LevenbergMarquardt minimization method can be realized in the following way. We shall put $m_{\nu}, \Omega_{b}, h$ and $b_{c l}$ or part of them free and find the minimum of $\chi^{2}$ for $N_{\nu}=1,2,3$ in a series. The lowest value from them will be suggested as minimum of $\chi^{2}$ for each set of free parameters. This is because the $N_{\nu}$ possesses the discrete value.

The key point is narrowing the range of search parameter values. The analytical approximation of the MDM power spectra used here is accurate enough in the following range of parameters: $0.3 \leq h \leq 0.7, \Omega_{\nu} \leq 0.5$, $\Omega_{b} \leq 0.3, N_{\nu} \leq 3$ [33]. By the upper and lower boundaries of $h, \Omega_{\nu}$ and $\Omega_{b}$ availability of the used analytical approximation we admeasure the range of search values of these parameters. We make these boundaries as 'mirror walls'.

\section{A. All parameters are free}

The minima of $\chi^{2}$ in a 4-dimensional space of parameters $\Omega_{\nu}, \Omega_{b}, h$ and $b_{c l}$ for models with 1,2 and 3 species of massive neutrinos are achieved for the set of parameters presented in table 2 . The spectra for them are shown in Fig. 5 and $\sigma_{8}$ 's are presented in the table 2. (The accuracy of analytical approximation of MDM spectra is better than $5 \%$ ).

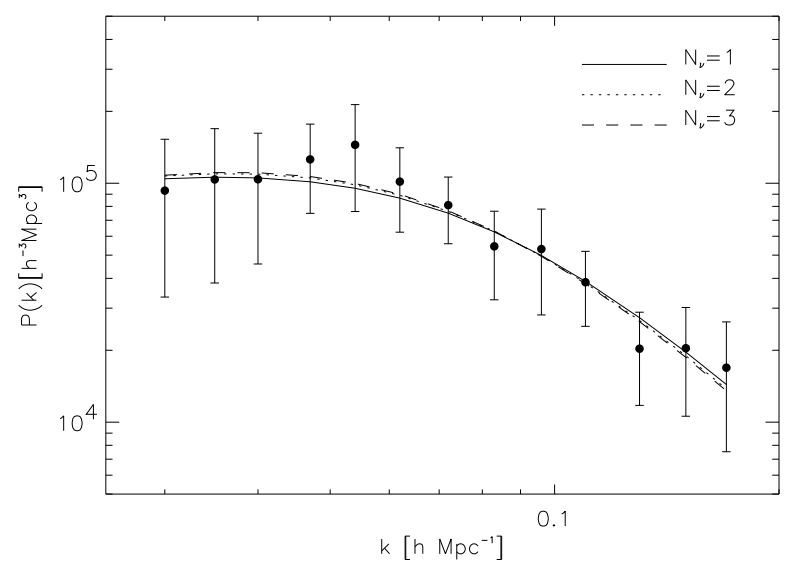

Fig. 5. The rich cluster power spectra of MDM models with best-fit parameters $\Omega_{\nu}, \Omega_{b}, h$ and $b_{c l}$ for 1,2 and 3 sorts of massive neutrinos (table 2). The filed circles are experimental Abell-ACO power spectrum by Retzlaff et al. 1997.

As we can see $\chi^{2}$ is few times lower than the formal degree of freedom, $d=n-m$, where $n$ is the number of data points, $m$ is the number of free parameters. The reason is that not all the points of the Abell-ACO power spectrum presented in table 1 are independent. The numerical experiment has shown that the minimal number of points which determine the same MDM parameters is $\approx 7$ (odd points of $P_{A}\left(k_{i}\right)$ in table 1 , for example). Indeed, such a spectrum can be described by amplitude and inclination at small and large scale ranges and the second order curve at the peak (or maximum) range. It means that real $d \approx 3-4$.

Therefore, in the 5 -dimension space of free parameters $\left(\Omega_{\nu}, N_{\nu}, \Omega_{b}, h\right.$ and $\left.b_{c l}\right)$ the global minimum of $\chi^{2}$ is achieved for the MDM model with 3 sorts of massive neutrinos. It has the lowest $m_{\nu}$ and the highest $h$ which better matches the data on immediate measurements of Hubble constant. However, it is unexpected that the found $\Omega_{\nu}$ is so high and $\Omega_{b}$ is so low. They contradict the data on high redshift objects and nucleosynthesis constraint $\left(0.007 \leq \Omega_{b} h^{2} \leq 0.024\right.$, [46,45,42]) accordingly. The MDM models with so high a $\Omega_{\nu}(\sim 0.4-0.5)$ also have a problem with the galaxy formation, $\sigma_{0} \sim 1$ for them. Let's analyze the cases with additional constraints which can lead us out of this difficulty. 


\section{B. Coordination with nucleosynthesis constraint}

The increasing of baryon content can decrease this difficulty (see [13]). We shall fix baryon content by the upper limit which is resulted from the nucleosynthesis constraint $\Omega_{b} h^{2}=0.024$ and keep up the rest parameters as free. The found best-fit parameters are in the table 3 , rich power spectrum for the case with 3 sorts of massive neutrino is shown in Fig. 6 (dotted line). The spectra for the cases with 1 and 2 sorts are close to this one.

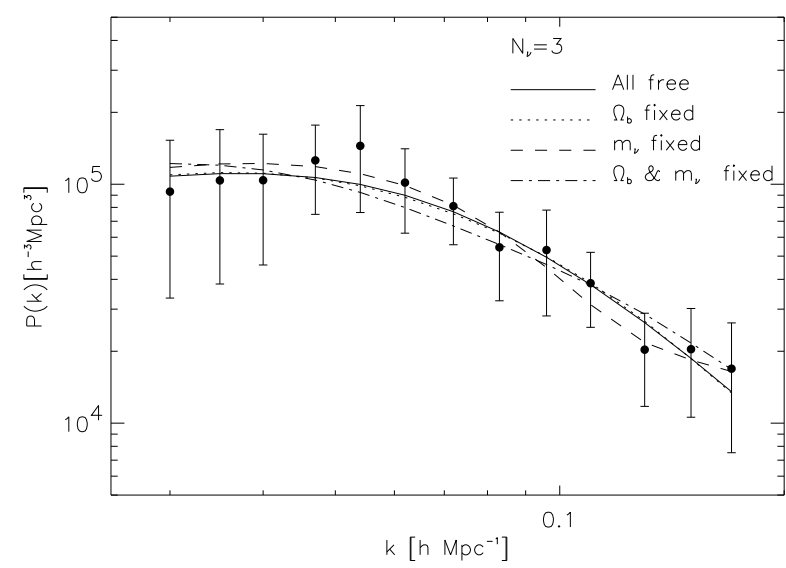

Fig. 6. The rich cluster power spectrum of MDM models with 3 sorts of massive neutrinos and best-fit parameters for the cases when all parameters are free (solid line), when baryon content $\Omega_{b}$ is fixed by nucleosynthesis constraint (dotted line), when mass of neutrino $m_{\nu}=2.5 \mathrm{eV}$ is fixed (dashed line) and when both $\Omega_{b}$ and $m_{\nu}$ are fixed (dashed dotted line). The filed circles are the same as in Fig. 5.

\begin{tabular}{|ccccccc|}
\hline$N_{\nu}$ & $\chi^{2}$ & $\Omega_{\nu}\left(m_{\nu}\right)$ & $\Omega_{b}$ & $h$ & $b_{c l}$ & $\sigma_{8}$ \\
\hline 1 & 2.58 & $0.42(8.3)$ & 0.12 & 0.46 & 3.56 & 0.54 \\
2 & 2.02 & $0.46(6.5)$ & 0.08 & 0.55 & 3.37 & 0.56 \\
3 & 1.82 & $0.48(5.7)$ & 0.06 & 0.62 & 3.27 & 0.57 \\
\hline
\end{tabular}

Table 3. Best-fit parameters of MDM models with 1, 2 and 3 sorts of massive neutrinos for Abell-ACO power spectrum by Retzlaff et al. 1997 and mass function by Girardi et al. 1998 when baryon content is fixed by nucleosynthesis constraint $\left(\Omega_{b} h^{2}=0.024\right)$.

As we can see $\Omega_{\nu}$ increases when $\Omega_{b}$ decreases and the minima of $\chi^{2}$ are achieved at high $\Omega_{\nu}$ again. But they are quite close to the corresponding minima from the previous table $\left(\Delta \chi^{2}<1\right)$.

\section{When the mass of neutrino is known}

An interesting question ensuing from last two items is: which best-fit values of $\Omega_{b}$ and $h$ can be obtained from these data on the Abell-ACO power spectrum and mass function in the case when mass of neutrino is determined by any physical or astrophysical experiments and is known. Let's assume that $m_{\nu}$ is fixed but the number of species $N_{\nu}$ is unknown. We fix $\Omega_{\nu}$ by relation (4) and the rest of parameters leave free. The search in such an approach was unsuccessful because it halted in the upper limit of $\Omega_{b}=0.3$. When this 'mirror wall' was removed the solutions were found but with extremely high content of baryons for which an accuracy of analytical approximation for MDM spectra is worse $(\sim 15-20 \%)$. Results for $m_{\nu}=2.5 \mathrm{eV}$ and $3 \mathrm{eV}$ are presented in table 4 . The rich cluster power spectrum for $m_{\nu}=2.5 \mathrm{eV}$ and $N_{\nu}=3$ is shown in Fig. 6 (dashed line). The spectra for 1 and 2 sorts are close to this one.

\begin{tabular}{|ccccccc|}
\hline$N_{\nu}$ & $\chi^{2}$ & $\Omega_{\nu}\left(m_{\nu}\right)$ & $\Omega_{b}$ & $h$ & $b_{c l}$ & $\sigma_{8}$ \\
\hline 1 & 1.53 & $0.05(2.5)$ & 0.47 & 0.75 & 3.22 & 0.65 \\
2 & 1.44 & $0.09(2.5)$ & 0.45 & 0.79 & 3.21 & 0.65 \\
3 & 1.39 & $0.12(2.5)$ & 0.43 & 0.82 & 3.20 & 0.65 \\
1 & 1.51 & $0.06(3.0)$ & 0.47 & 0.75 & 3.21 & 0.65 \\
2 & 1.42 & $0.10(3.0)$ & 0.44 & 0.79 & 3.20 & 0.65 \\
3 & 1.37 & $0.14(3.0)$ & 0.42 & 0.83 & 3.19 & 0.65 \\
\hline
\end{tabular}

Table 4. Best-fit parameters of MDM models with 1, 2 and 3 sorts of massive neutrinos for Abell-ACO power spectrum by Retzlaff et al. 1997 and mass function by Girardi et al. 1998 when neutrino mass is fixed $\left(m_{\nu}=2.5\right.$ and $3.0 \mathrm{eV}$, $\Omega_{\nu}=m_{\nu} N_{\nu} / 93 h^{2}$ ).

The $\chi^{2}$ 's in all cases here are lower than in table 2 because the performance of analytical approximation of MDM spectra for so high a $\Omega_{b}$ and $h$ is essentially worse than in the allowance range. Therefore we can not conclude that the global minimum of $\chi^{2}$ in the 4 -dimension space of parameters $m_{\nu}, N_{\nu}, \Omega_{b}$ and $h$ is in the range of high $\Omega_{b}$ and $h$. It is in point with the parameters which are in the last row of table 2. But we certainly conclude that when $m_{\nu} \sim 2-3 \mathrm{eV}$ the minimum is absent in the range of $\Omega_{b} \leq 0.3,0.3 \leq h \leq 0.7$. Therefore the Abell-ACO power spectrum and mass function among the MDM models with $m_{\nu} \leq 4 \mathrm{eV}$ and $N_{\nu} \leq 3$ prefer $\Omega_{b}>0.3$ and $h \sim 0.8$ that agrees well with the results by $[13]$.

\section{D. $\Omega_{b}$ and $m_{\nu}$ are fixed}

One can look now which $h$ is preferable by AbellACO power spectrum and mass function when neutrino mass and baryon content are fixed by the other observable constraints or theoretical arguments. Let's put that $m_{\nu}=2.5 \mathrm{eV}\left(\Omega_{\nu}=m_{\nu} N_{\nu} / 93 h^{2}\right)$ and $\Omega_{b}=0.024 / h^{2}$ is fixed by the upper limit of nucleosynthesis constraint. Only $h$ and $b_{c l}$ are free parameters. Their best-fit values found for 1, 2 and 3 sorts of massive neutrino are presented in the table 5 . The rich cluster power spectrum for $N_{\nu}=3$ MDM model with those parameters is shown in Fig. 6 (dashed dotted line). The spectra for 1 and 2 sorts are close to this one. 


\section{B. NOVOSYADLYJ}

As we see in the MDM model with 3 sorts of $2.5 \mathrm{eV}$ neutrinos the best-fit value of $h$ and $\sigma_{8}$ are closer to the corresponding observable data than in models with 1 or 2 sorts.

\begin{tabular}{|ccccccc|}
\hline$N_{\nu}$ & $\chi^{2}$ & $\Omega_{\nu}\left(m_{\nu}\right)$ & $\Omega_{b}$ & $h$ & $b_{c l}$ & $\sigma_{8}$ \\
\hline 1 & 4.20 & $0.16(2.5)$ & 0.15 & 0.41 & 3.90 & 0.55 \\
2 & 3.31 & $0.24(2.5)$ & 0.11 & 0.47 & 3.76 & 0.56 \\
3 & 2.85 & $0.29(2.5)$ & 0.09 & 0.52 & 3.69 & 0.56 \\
\hline
\end{tabular}

Table 5. Best-fit parameters of MDM models with 1, 2 and 3 sorts of massive neutrinos for Abell-ACO power spectrum by Retzlaff et al. 1997 and mass function by Girardi et al. 1998 when baryon content and neutrino mass are fixed: $\Omega_{b}=0.024 / h^{2}, m_{\nu}=2.5 \mathrm{eV}\left(\Omega_{\nu}=m_{\nu} N_{\nu} / 93 h^{2}\right)$.

\section{DISCUSSION}

Rich cluster power spectra of models with the best fit parameters are within the error bars of the corresponding experimental data (Fig. 5-6). But none of them explains the peak at $k \approx 0.05 h / M p c$ that corresponds to the linear scale $\approx 120 h^{-1} \mathrm{Mpc}$. It has excess power at $\sim 50 \%$ in comparison with the best-fit model and $\sim 30 \%$ in comparison with the high $-\Omega_{b}$ one. It is more prominent yet in the data by [10]. Apparently, it is a real feature of the power spectrum. The necessity of a similar feature in the power spectrum was argued earlier by the explanation of Great Attractor phenomenon [19,32]. A sample of the Abell-ACO clusters of galaxies used by [40] is placed in $60^{\circ}$ double-cone with the axis pointing towards the Milky Way pole. The Great Attractor, on the contrary, placed in the plane of our galaxy. Therefore, they are an independent experimental demonstration of the reality of those peak. Other important arguments for its validity come from pencil-beam redshift survey by [5] and from 2-dimensional power spectrum of the Las Campanas Redshift Survey [25]. The angular correlations in the APM survey [15] and high-redshift absorption lines in quasar spectrum [39] also show similar features at these scales. It was shown also by [1] that this $\sim 120 h^{-1} \mathrm{Mpc}$ peak well agree with Saskatoon data on the $\Delta T / T$ power spectrum. Therefore, the data used here on rich cluster power spectrum are based on the surveys which represent a fair sample of $\sim 120 h^{-1} \mathrm{Mpc}$ structures and that peak is significant despite the large error bars of experimental data.

Obviously, that turnabout to open $\left(\Omega_{0}<1\right)$ models or flat with cosmological term $\left(\Omega_{0}+\Omega_{\Lambda}=1\right)$ does not improve the situation with the explanation of that peak in our approach. It is because the maximum of power spectra in those models is shifted to larger scales in comparison with matter dominated flat models analyzed here. Explaning of it by baryonic acoustic oscillations calls for extremely high content of baryons that disagree with nucleosynthesis constraint (see [13]). Therefore we face a necessity to consider models with a built-in scale in the primordial power spectrum again.
Let's determine the parameters of this peak. The comparison of rich cluster power spectrum predicted by the MDM model with the best-fit parameters (table 2) with the observable one showed that the peak has approximately the Gaussian form. Therefore we approximate it by the function $p(k)=1+$ $a_{p} \exp \left(2\left(k_{p}-k\right)^{2} / w_{p}^{2}\right)$, where $a_{p}, k_{p}$ and $w_{p}$ are amplitude, center and width of the peak accordingly. We set the power spectrum in the form of $P_{M D M+p}(k)=$ $P_{M D M}\left(k ; \Omega_{b}, h, m_{\nu}, N_{\nu}\right) p\left(k ; a_{p}, k_{p}, w_{p}\right)$, and repeat previous calculations with additional free parameters $a_{p}, k_{p}$ and $w_{p}$.

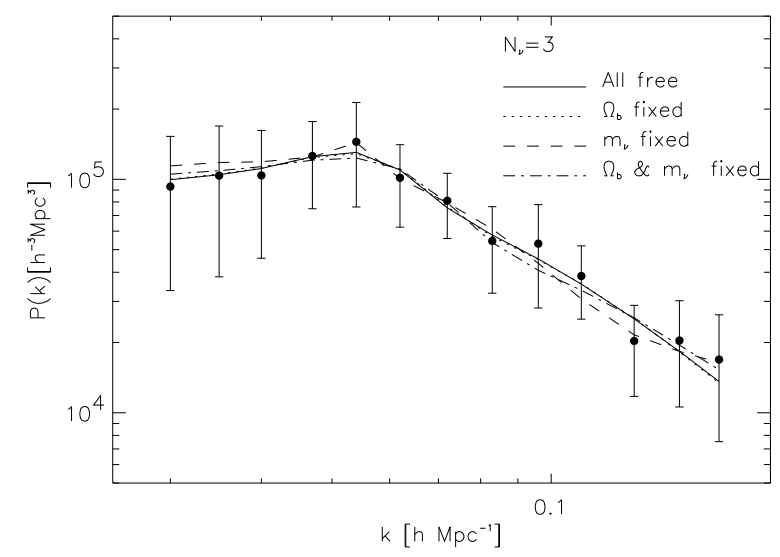

Fig. 7. The rich cluster power spectrum of MDM+peak models with 3 sorts of massive neutrino and best-fit parameters from table 6. The filed circles are the same as in Fig. 5.

\begin{tabular}{|llccccc|}
\hline$m_{\nu}$ & $\Omega_{b}$ & $h$ & $b_{c l}$ & $k_{p}$ & $a_{p}$ & $w_{p}$ \\
\hline 4.6 & 0.01 & 0.58 & 3.14 & 0.056 & 0.46 & 0.011 \\
5.0 & $0.064^{(*)}$ & 0.61 & 3.11 & 0.056 & 0.47 & 0.012 \\
$2.5^{(*)}$ & 0.424 & 0.82 & 3.16 & 0.054 & 0.34 & 0.007 \\
$2.5^{(*)}$ & $0.084^{(*)}$ & 0.53 & 3.33 & 0.060 & 0.63 & 0.013 \\
\hline
\end{tabular}

Table 6. Best-fit parameters of MDM+peak models with 3 sorts of massive neutrinos for Abell-ACO power spectrum by Retzlaff et al. 1997 and mass function by Girardi et al. 1998. The fixed parameters are noted by ${ }^{(*)}\left(\Omega_{b}=0.024 / h^{2}\right.$, $\left.m_{\nu}=2.5 \mathrm{eV}\right)$.

It should seem that this peak causes such high best-fit values of $\Omega_{\nu}$ or $\Omega_{b}$ in tables $2-4$. The results of the search for best-fit parameters in the 8 -dimensional space of the MDM+peak model parameters showed that it is not so, that well agrees with the numerical results by [40]. The introducing of the peak really decreases the $\chi^{2}$ but the MDM model parameters are changed weakly. It is because they are determined mainly by the inclination of the Abell-ACO power spectrum after the peak and $\tilde{\sigma}_{8}$ as the most accurate value of the data set used here. The models with 3 sorts of massive neutrino are preferable like in the previous cases. In table 9 the best-fit parameters of the MDM models with 3 sorts of massive neutrino 
as well as best-fit parameters of the peak are presented for 4 cases: all the MDM parameters were free (1st row), baryon content $\Omega_{b}$ was fixed by the upper limit of nucleosynthesis constraint (2), neutrino mass was fixed at $m_{\nu}=2.5 \mathrm{eV}(3), \Omega_{b}$ and $m_{\nu}$ were fixed (4). The $\chi^{2}$ for them are $0.81,0.86,1.11,1.04$ accordingly. In all the cases except $(3)$ the $\sigma_{8}=0.6$, in (3) case the $\sigma_{8}=0.66$. The rich cluster power spectrum for these cases are shown in Fig. 7.

The introducing of such a peak increases the predicted bulk velocities in a top-hat sphere of the radius $R$ whose r.m.s. values can be calculated according to

$$
V_{R}^{2}=\frac{H_{0}^{2}}{2 \pi^{2}} \int_{0}^{\infty} d k P_{M D M}(k) W^{2}(k R),
$$

where $W(k R)$ is the Fourier transform of this sphere. So, for $R=50 h^{-1} \mathrm{Mpc}$ it increases from $340 \mathrm{~km} / \mathrm{s}$ to $360 \mathrm{~km} / \mathrm{s}$ for the best-fit model (3rd row of table $2,1 \mathrm{st}$ row of table 6) and from $330 \mathrm{~km} / \mathrm{s}$ to $345 \mathrm{~km} / \mathrm{s}$ for a model with fixed $m_{\nu}$ and $\Omega_{b}$ (3rd row of table 5 , the last row of table 6 ). The observable value of bulk velocity for this scale is $\tilde{V}_{50}=375 \pm 85 \mathrm{~km} / \mathrm{s}$, which follows from Mark III POTENT results [21]. Therefore, this peak is preferable also by the data on large scale peculiar velocity of galaxies and Great Attractor like structures. However, the models with high values of $\Omega_{\nu} \sim 0.4-0.5\left(m_{\nu} \sim 4-\right.$ $7 \mathrm{eV}$ ), which are best-fit ones for the Abell-ACO data, have problems with the explanation of galaxy scale structures and high redshift objects. But models with median $\Omega_{\nu} \sim 0.2-0.3\left(m_{\nu} \sim 2.5, N_{\nu} \sim 2-3\right)$ are not ruled out by these data $\left(\Delta \chi^{2}<1\right)$. On the contrary, the CDM model with $\Omega_{b} \leq 0.2$ and $h>0.5$ is ruled out by these data at a high confidence level because for them $\Delta \chi^{2} \geq 15$.

At last it must be noted that primordial spectrum feature like this peak is inherent for double inflation models $[23,22,24,18,36]$ and inflationary model wherein an inflation field evolves through a kink in the potential [47]. Both classes of these models were confronted with the observational data on the Abell-ACO power spectrum by [26] and [40] accordingly.

\section{CONCLUSIONS}

The Abell-ACO power spectrum by [40] and mass function by [16] in the parameter space of the MDM model $\left(\Omega_{0}=1\right)$ prefer a region with high $\Omega_{\nu}(\sim 0.4-0.5)$, low $\Omega_{b}(\leq 0.01)$ and $h(\sim 0.4-0.6)$. The best-fit parameters are as follows: $N_{\nu}=3, m_{\nu}=4.4 \mathrm{eV}, h=0.56$, $\Omega_{b} \leq 0.01$. Unfortunately, experimental uncertainties of the data used here for the determination of these parameters give no chance to rule out models with a different set of parameters at a sufficiently high confidence level. The MDM models with baryon content at the upper limit of the nucleosynthesis constraint $\left(\Omega_{b} h^{2}=0.024\right)$ do not outstep $\Delta \chi^{2}=1$ of best-fit model (see table 3 ). The high- $\Omega_{b}(\sim 0.4-0.5)$ solutions are obtained when neutrino mass are fixed and $\leq 3 \mathrm{eV}$.

Introducing artificially into the primordial power spectrum a peak of Gaussian form decreases the $\chi^{2}$, increases the bulk motions but does not change essentially the best-fit parameters of the MDM models. It means that determinative for these parameters is mainly inclination of the Abell-ACO power spectrum at the scales smaller than the scale of the peak position and $\tilde{\sigma}_{8}$ as the most accurate value of the data set used here.

Hereby, the power spectrum of the Abell-ACO clusters of galaxies and mass function are a sensitive test for the MDM model parameters. But more accurate data on power spectrum of matter density fluctuations are necessary for more certain determination of cosmological parameters.

\section{ACKNOWLEDGMENTS}

This work was performed thanks to the financial support granted by Swiss National Science Foundation (grant NSF 7IP050163) and DAAD in Germany (Ref.325). The author also thanks AIP for hospitality and S. Gottloeber for useful discussions.
[1] F. Atrio-Barandela, J. Einasto, S. Gottloeber, V. Muller, A. Starobinsky, JETP Lett. 66, 397 (1997).

[2] C. L. Bennett et al., Astrophys. J. 436, 423 (1994).

[3] C. L. Bennett et al, Astrophys. J. Lett. 464, 1 (1996).

[4] Z. G. Berezhiani, A. D. Dolgov, R. N. Mohapatra, preprint hep-ph/9511221, 1995.

[5] T. J. Broadhurst, R. S. Ellis, D. C. Koo, A. S. Szalay, Nature (London) 343, 726 (1990).

[6] E. F. Bunn, M. White, Astrophys. J. 480, 6 (1997).

[7] M. Davis, F. J. Summers, D. Schlegel, Nature (London) 359, 393 (1992).

[8] S. Dodelson, E. Gates, A. Stebbins, Astrophys. J. 467, 10 (1996).

[9] R. Durrer, M. Kunz, C. Lineweaver, M. Sakellariadou, preprint astro-ph/9706215, 1997.

[10] J. Einasto, M. Einasto, S. Gottloeber, V. Muller, V. Saar,
A. A. Starobinsky, E. Tago, D. Tucker, H. Andernach, Nature (London), 385, 139 (1997).

[11] D. J. Eisenstein, W. Hu, preprint astro-ph/9709112, 1997.

[12] D. J. Eisenstein, W. Hu, preprint astro-ph/9710252, 1997.

[13] D. J. Eisenstein, W. Hu , J. Silk , A. S. Szalay, preprint astro-ph/9710303, 1997.

[14] Y. Fukuda et al., Phys. Rev. Lett. 81, 1562 (1998).

[15] E. Gaztanaga, C. M. Baugh, preprint astro-ph/9704246, 1997.

[16] M. Girardi, S. Borgani, G. Giuricin, F. Mardirossian, M. Mezzetti, preprint astro-ph/9804188, 1998.

[17] Jeans scale for neutrino component in all cases analysed here is smaller than the cluster scales therefore all the 


\title{
B. NOVOSYADLYJ
}

matter is clustered and the term $\Omega^{0.6}$ in the original form is omited

[18] S. Gottloeber, V. Muller, A. A. Starobinsky, Phys. Rev. D 43, 2510 (1991)

[19] B. I. Hnatyk, V. N. Lukash, B. S. Novosyadlyj, Astron. Astrophys., 300, 1 (1995).

[20] J. A. Holtzman, Astrophys. J. Suppl. Ser. 71, 1 (1989).

[21] T. Kolatt, A. Dekel, Astrophys. J. 479, 592 (1997).

[22] L. A. Kofman, A. D. Linde, Nucl. Phys. B 282, 555 (1987).

[23] L. A. Kofman, A. D. Linde, A. A. Starobinsky, Phys. Lett. B 157, 361 (1985).

[24] L. A. Kofman, D. Pogosyan, Phys. Lett. B 214, 508 (1988).

[25] S. D. Landy, S. A. Shectman, H. Lin et al., Astrophys. J. Lett. 456, 1 (1996).

[26] J. Lesgourgues, D. Polarski, A. A. Starobinsky, preprint astro-ph/9711139, 1997.

[27] A. R. Liddle, D. H. Lyth , P. T. P. Viana, M. White, Mon. Not. R. Astron. Soc. 282, 281 (1996).

[28] C. A. Lineweaver, D. Barbosa, preprint astroph/9706077, 1997.

[29] C.-P. Ma, Astrophys. J. 471, 13 (1996); preprint astroph/9605198, 1996.

[30] C. B. Netterfield, M. J. Devlin, N. Jarosik et al., Astrophys. J. 474, 47 (1997).

[31] B. Novosyadlyj, Kinematics Phys. Celest. Bodies 10, 7 (1994).

[32] B. Novosyadlyj, Astron. Astrophys. 10, 85 (1996).

[33] B. Novosyadlyj, R. Durrer, V. N. Lukash, preprint astroph/9811262, 1998; Astron. Astrophys. Trans. 1999 (in press).

[34] J. A. Peacock, S. J. Dodds, Mon. Not. R. Astron. Soc.
267, 1020 (1994).

[35] D. Yu. Pogosyan, A. A. Starobinsky, Astrophys. J. 447, 465 (1994).

[36] D. Polarski, A. A. Starobinsky, Nucl. Phys. D 35, 419 (1992).

[37] W. H. Press, B. P. Flannery, S. A. Teukolsky, W. T. Vettrling Numerical recipes in FORTRAN (Cambridge Univ. Press, New York, 1992).

[38] J. R. Primak, M. A. Gross, preprint astro-ph/981024, 1998.

[39] J. M. Quashnock, D. E. Vanden Berg, D. G. York, Astrophys. J. Lett. 472, 69 (1996).

[40] J. Retzlaff, S. Borgani, S. Gottloeber, A. Klypin, V. Muller, preprint astro-ph/9709044, 1997.

[41] R. K. Schaefer, Q. Shafi, Nature 359, 199 (1992).

[42] D. Schramm, M. Turner, preprint astro-ph/9706069, (1997).

[43] U. Seljak, M. Zaldarriaga, Astrophys. J. 469, 437 (1996); preprint astro-ph/9603033, 1996.

[44] G. F. Smoot, C. L. Bennett, A. Kogut et al., Astrophys. J. Lett. 396, 1 (1992).

[45] A. Songaila, E. Wampler, L. L. Cowie, Nature, 385, 137 (1997).

[46] D. Tytler, X. M. Fan, S. Burles, Nature 381, 207 (1996).

[47] A. A. Starobinsky, JETP Lett. 55, 489 (1992).

[48] R. Valdarnini, T. Kahniashvili, B. Novosyadlyj, Astron. Astrophys. 336, 11 (1998); preprint astro-ph/9804057, 1998.

[49] A. Van Dalen, R. K. Schaefer, Astrophys. J. 398, 33 (1992).

[50] P. T. P. Viana, A. W. Liddle, Mon. Not. R. Astron. Soc. 281, 323 (1996).

\section{ОПТИМАЛЬНІ ПАРАМЕТРИ МОДЕЛ І ЗМІШАНОЇ ТЕМНОЇ МАТЕРІЇ, ЯКІ ВИПЛ ИВАЮТЬ ІЗ СПЕКТРА ПОТУ ЖНОСТИ І ФУНКЦІЇ МАС ABЕLL-ACO СКУПЧЕНЬ ГАЛ АКТИК}

\author{
Б. Новося длий \\ Астрономічна обсерваторія Львібського державного університету імені Івана Франка, \\ вул. Кирила і Мефодія, 8, Львів, 290005, Украӥна
}

\begin{abstract}
Проаналізовано можливість визначення параметрів моделі змішаної темної матерії (ЗТМ) на основі спостере жу ваних даних про спектр поту жности збурень густини речовини, який виведений із просторового розподілу скупчень галактик в Abell-ACO каталозі та їньої функції мас. Показано, що область спектра, яка відповідає цим показникам, $є$ достатньо чутливою до таких параметрів моделі ЗТ М, як маса спокою нейтрино $m_{\nu}$, число сортів масивних нейтрино $N_{\nu}$, вміст баріонів $\Omega_{b}$ і постійна Хаббла $h \equiv H_{0} / 100 \mathrm{~km} / \mathrm{s} / \mathrm{Mpc}$. Для іх визначення застосовано метод $\chi^{2}$ мінімізації. Якщо всі ці параметри є вільними, тобто підлягають визначенню, тоді ці експериментальні дані виділяють область із високим $\Omega_{\nu}(\sim 0.4-0.5)$, низьким $\Omega_{b}$ $(<0.01)$ і $h(\sim 0.4-0.6)$. Мінімуму $\chi^{2}$ досягають при таких параметрах: $N_{\nu}=3, m_{\nu}=4.4 \mathrm{eV}, h=0.56$, $\Omega_{b} \leq 0.01$. Розв'язки з високими значеннями $\Omega_{b} \sim 0.4-0.5$ отримують, якщо маса нейтрино $\epsilon$ фіксованою $\mathrm{i}$ $\leq 3 \mathrm{eV}$.

Для пояснення спостере жуваного надлишку потужности на $k \approx 0.05 h / M p c$ у первинний спектр було введено пік у формі гаусіяна. Його параметри (амплітуду, положення і ширину) визначали разом із параметрами моделі ЗТМ. Він зменшує мінімальне значення $\chi^{2}$, збільшує величину пвидкости об'ємного потоку галактик, але не змінює суттєво параметрів моделі ЗТ М, які відповідають мінімуму $\chi^{2}$.

Також показано, що моделі з промі жними значеннями $\Omega_{\nu}(\sim 0.2-0.3)\left(m_{\nu} \sim 2.5, N_{\nu} \sim 2-3\right)$ і $\Omega_{b}=$ $0.024 / h^{2}$, яке випливає 3 даних космологічного нуклеосинтезу і об'єктів на високих червоних зміщеннях, цими даними не заперечуються $\left(\Delta \chi^{2}<1\right)$.
\end{abstract}

